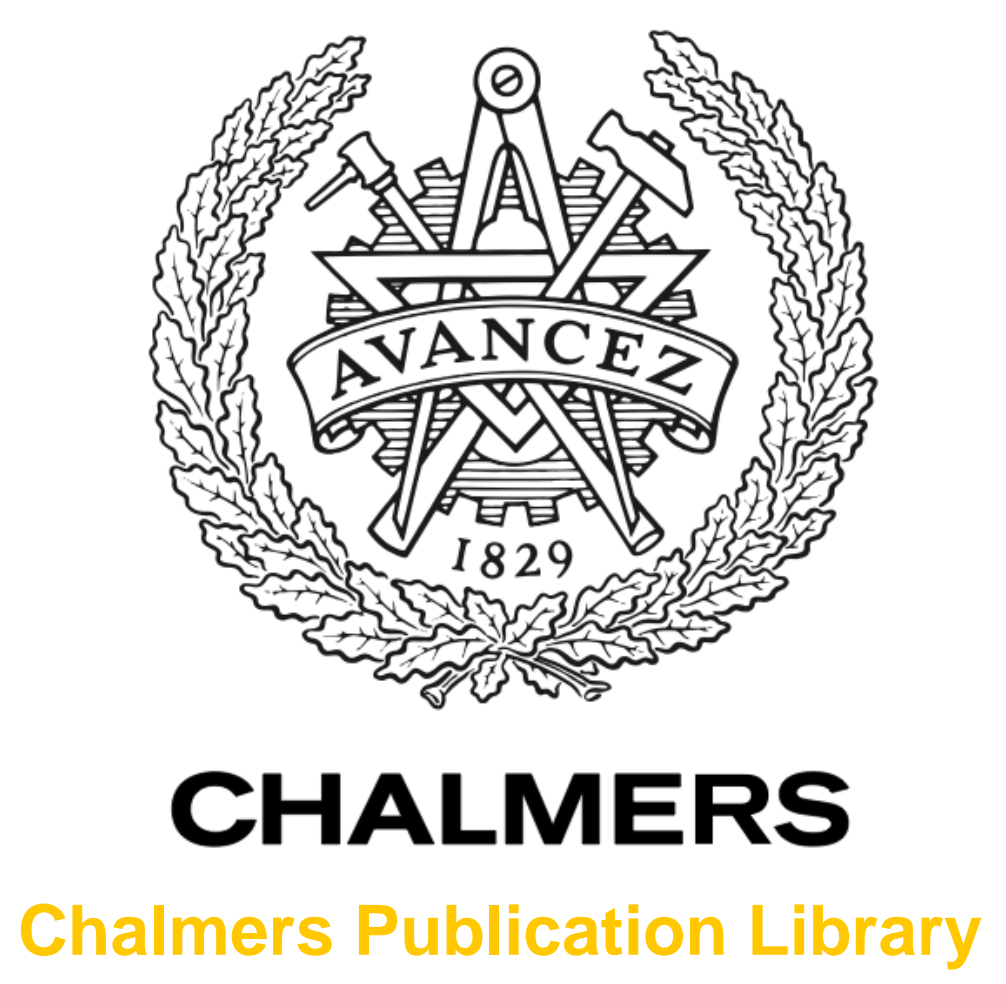

Design of Maximally Sparse Antenna Arrays in the Presence of Mutual Coupling

This document has been downloaded from Chalmers Publication Library (CPL). It is the author's version of a work that was accepted for publication in:

IEEE Antennas and Wireless Propagation Letters (ISSN: 1536-1225)

Citation for the published paper:

Bencivenni, C. ; Ivashina, M. ; Maaskant, R. et al. (2015) "Design of Maximally Sparse Antenna Arrays in the Presence of Mutual Coupling". IEEE Antennas and Wireless

Propagation Letters, vol. 14 pp. 159-162.

http://dx.doi.org/10.1109/lawp.2014.2357450

Downloaded from: http://publications.lib.chalmers.se/publication/214502

Notice: Changes introduced as a result of publishing processes such as copy-editing and formatting may not be reflected in this document. For a definitive version of this work, please refer to the published source. Please note that access to the published version might require a subscription.

Chalmers Publication Library (CPL) offers the possibility of retrieving research publications produced at Chalmers University of Technology. It covers all types of publications: articles, dissertations, licentiate theses, masters theses, conference papers, reports etc. Since 2006 it is the official tool for Chalmers official publication statistics. To ensure that Chalmers research results are disseminated as widely as possible, an Open Access Policy has been adopted.

The CPL service is administrated and maintained by Chalmers Library. 


\title{
Design of Maximally Sparse Antenna Arrays in the Presence of Mutual Coupling
}

\author{
C. Bencivenni, M. V. Ivashina, Senior Member, IEEE, R. Maaskant, Senior Member, IEEE, and J. Wettergren
}

\begin{abstract}
An optimization framework is proposed that combines electromagnetic field simulations with an iterative $\ell_{1}$-norm minimization procedure to synthesize optimally sparse antenna arrays. The proposed approach overcomes the limitation of design methodologies that assume idealized antenna elements employing equal (isolated or isotropic) element patterns, as these yield suboptimal solutions when the antenna mutual coupling effects cannot be ignored. The latter is demonstrated for arrays of relatively strongly coupled dipole radiators.
\end{abstract}

Index Terms-sparse array antennas, aperiodic array, thinning, method of moments, mutual coupling.

\section{INTRODUCTION}

A NTENNA arrays have the potential to meet the stringent requirements on the multi-beam, multi-channel, and beam reconfigurability in many future communication and sensor systems. However, a critical requirement is to minimize the number of array elements filling the antenna aperture in order to reduce the electronic beamforming cost, the power consumption, and overall design complexity.

Aperiodic arrays, as first introduced by Unz [1], are potentially capable to address the above-mentioned requirements. To design such arrays, two approaches are commonly used: (i) the Sparse Array approach, where a minimum number of elements is optimally positioned according to a specified criterion [2]; and (ii) the Thinned Array approach, where the optimization starts from a densely filled regular array, after which some elements are removed to obtain an aperiodic array layout while meeting certain specifications [3]. Several ways of implementing these approaches have been proposed, which are based on stochastic, analytical, or deterministic methods. Stochastic methods, such as e.g. genetic algorithms, are used as global optimizers (GO) in the array synthesis problems [4]; however, they typically cannot handle large arrays but may be well-suited to refine an initial solution [5]. Examples of analytical methods are the Matrix Pencil Method [6], the orthogonalization method, and Almost Different Sets [3]. A popular deterministic approach is to position elements on a nonuniform grid whose density is proportional to a reference continuous aperture field distribution, such as the Taylor taper [7], but this may result in a (too) large number of elements.

C. Bencivenni, M. V. Ivashina, and R. Maaskant are with the Signals and Systems Department of the Chalmers University of Technology, Göteborg, Sweden, e-mail: carlo.bencivenni@chalmers.se, marianna.ivashina@chalmers.se, rob.maaskant@chalmers.se. J. Wettergren is with RUAG Space AB, Göteborg, Sweden, johan.wettergren@ruag.com.

This work is financed by Swedish VR and VINNOVA grants.

Manuscript received April xx, 20xx; revised January xx, 20xx.
In these methods, one commonly considers idealized elements (e.g. isolated or isotropic), which may be inadequate in some cases as they neglect important electromagnetic (EM) effects, such as the antenna mutual coupling (MC). The beam degradation due to $\mathrm{MC}$ effects can be quantified by means of a posteriori full-wave simulations, as in [8], or corrected for through the multiplication of the steering vector by the antenna MC matrix to obtain the effective steering vector as in [9]; the latter is an array signal processing (ASP) method for estimating the direction of arrival in the presence of known mutual coupling effects, but is limited to minimum scattering antennas [10]. However, to the author's best knowledge, no optimization framework exists that combines EM field simulations with ASP techniques during the array synthesis phase to yield a sparse array solution of mutually coupled antenna elements without resorting to GO techniques [11]. In this paper we propose to hybridize the recently introduced iterative $\ell_{1}$-norm minimization procedure as the array sparsification algorithm [12] with full-wave MoM simulations.

The paper is organized as follows. In Sec. II the $\ell_{1}$ convex optimization procedure for thinning the array is described along with the newly proposed full-wave antenna array optimization framework. The numerical results are described in Sec. III, where a small and large linear array of relatively strongly coupled dipole radiators is considered. It is shown that the solution is more optimal if one accounts for the $\mathrm{MC}$ effects as opposed to ignoring them.

\section{Methodology}

The minimization of the number of array elements is a nonconvex optimization problem, but may be reformulated as a tractable iterative convex optimization problem as described in [12]. The presently proposed optimization framework is based on the latter iterative approach, which will be summarized in the next sub-section for completeness, albeit slightly revisited to introduce the $\mathrm{MC}$-dependent vector element patterns, while the second sub-section deals with the integration of a full wave solver.

\section{A. Optimally Sparse Arrays}

Consider an array of $N$ elements placed at the arbitrary locations $\left\{\boldsymbol{r}_{n}\right\}_{n=1}^{N}$ along with the corresponding set of embedded far-field vector element patterns $\left\{\boldsymbol{f}_{n}(\hat{\boldsymbol{r}})\right\}_{n=1}^{N}$, where the direction $\hat{\boldsymbol{r}}(\theta, \phi)=\sin (\theta) \cos (\phi) \hat{\boldsymbol{x}}+\sin (\theta) \sin (\phi) \hat{\boldsymbol{y}}+\cos (\theta) \hat{\boldsymbol{z}}$. Hence, the resulting array far-field function can be written as

$$
\boldsymbol{f}(\hat{\boldsymbol{r}})=\sum_{n=1}^{N} w_{n} \boldsymbol{f}_{n}(\hat{\boldsymbol{r}}) \quad \text { with } \quad \boldsymbol{f}_{n}(\hat{\boldsymbol{r}})=\boldsymbol{f}_{n}^{0}(\hat{\boldsymbol{r}}) e^{j k \boldsymbol{r}_{n} \cdot \hat{\boldsymbol{r}}},
$$


where $w_{n}$ is the complex excitation coefficient of the $n$th element, and where $k$ is the wavenumber ${ }^{1}$. Note that $\boldsymbol{f}_{n}$ includes the propagation phase delay with respect to $f_{n}^{0}$, whose origin is on the element itself. Let the $N$-dimensional excitation vector $\mathbf{w}=\left[w_{1}, w_{2}, \ldots w_{N}\right]^{T}$, where ${ }^{T}$ denotes the transpose, and let us expand $\boldsymbol{f}=f_{\mathrm{co}} \hat{\mathbf{c o}}+f_{\mathrm{xp}} \mathbf{x p}$ into its far-field co-polar and cross-polar components, then Eq. (1) can be rewritten in the compact form, $\boldsymbol{f}(\hat{\boldsymbol{r}})=\left[\mathbf{w}^{T} \mathbf{f}_{\mathrm{co}}(\hat{\boldsymbol{r}})\right] \hat{\mathbf{c o}}+\left[\mathbf{w}^{T} \mathbf{f}_{\mathrm{xp}}(\hat{\boldsymbol{r}})\right] \hat{\mathrm{xp}}$ where $\mathbf{f}_{\nu}=\left[f_{\nu, 1}, f_{\nu, 2}, \ldots f_{\nu, N}\right]^{T}$ is an $N$-element column vector with $\nu \in\{\mathrm{co}, \mathrm{xp}\}$.

The problem of designing a maximally sparse array is finding $\mathbf{w}$ with a minimum number of non-zero entries while fulfilling certain pattern constraints. That is, solve the problem $\operatorname{argmin}\|\mathbf{w}\|_{\ell_{0}}$ with $\mathbf{w} \in \mathbb{C}^{N}$, subject to some pattern constraints. However, this non-convex problem cannot be solved in closed form, and finding a solution using a combinatorial search method is intractable, even for moderate array sizes.

In [12] the problem is relaxed and solved in a semi-analytic manner by approximating the $\ell_{0}$-norm minimization through an iterative weighted $\ell_{1}$-norm minimization. For a focused beam pattern in the scanning direction $\hat{\boldsymbol{r}}_{\mathrm{s}}$ with a prescribed side-lobe level (SLL) mask $M(\hat{\boldsymbol{r}})$ of the dominant polarization $\nu$, the $i$ th iteration of the algorithm reads [12]

$\underset{\mathbf{w}^{i} \in \mathbb{C}^{N}}{\operatorname{argmin}}\left\|\mathbf{Z}^{i} \mathbf{w}^{i}\right\|_{\ell_{1}}$, subject to $\left\{\begin{array}{l}f_{\nu}\left(\hat{\boldsymbol{r}}_{\mathrm{s}}\right)=1, \\ \left|f_{\nu}(\hat{\boldsymbol{r}})\right|^{2} \leq M(\hat{\boldsymbol{r}}), \hat{\boldsymbol{r}} \in \text { mask }\end{array}\right.$

where the $p$ th element of the diagonal matrix $\mathbf{Z}^{i}$ is given as $z_{p}^{i}=1 /\left(\left|w_{p}^{(i-1)}\right|+\epsilon\right)$ and is chosen to maximally enhance the sparsity of the solution $\mathbf{w}^{i}$; that is, redundant element are effectively suppressed through magnifying its apparent contribution in the minimization process by an amount that is based on the previous solution $\mathbf{w}^{(i-1)}$. The parameter $\epsilon$ enables elements that are "turned off" to be engaged again later on during the iterative procedure. It is recommended to set $\epsilon$ slightly smaller than the expected active excitations for an optimal convergence rate and stability. This numerically efficient procedure typically requires only few iterations for the excitation vector to converge.

\section{B. Inclusion of Mutual Coupling Effects}

The newly proposed array synthesis method involves two subsequent steps (see Fig. 1). First, the pattern mask is defined and the aperture is sampled finely enough to emulate a quasi-continuous element positioning (typical step size is $\Delta d=\lambda / 100$ ). Following [12], a maximally sparse array configuration is synthesized in the absence of MC effects, but where we assume phase-shifted versions of an EM-simulated isolated element pattern (IEP). The $\ell_{1}$-norm minimization is iterated till convergence, yielding the initial optimal element positions for the uncoupled case. Elements are deemed active or not based on a threshold level on the excitation magnitudes, since, typically, inactive elements have normalized magnitudes

\footnotetext{
${ }^{1}$ In [12] isolated (specifically isotropic) element patterns are considered, therefore ignoring MC. Due to the identical element patterns, the resulting far-field can be expressed as the product of the isolated element pattern times the array factor $\operatorname{AF}(\theta)=\sum_{n=1}^{N} w_{n} e^{j k \boldsymbol{r}_{n} \cdot \hat{\boldsymbol{r}}}$.
}

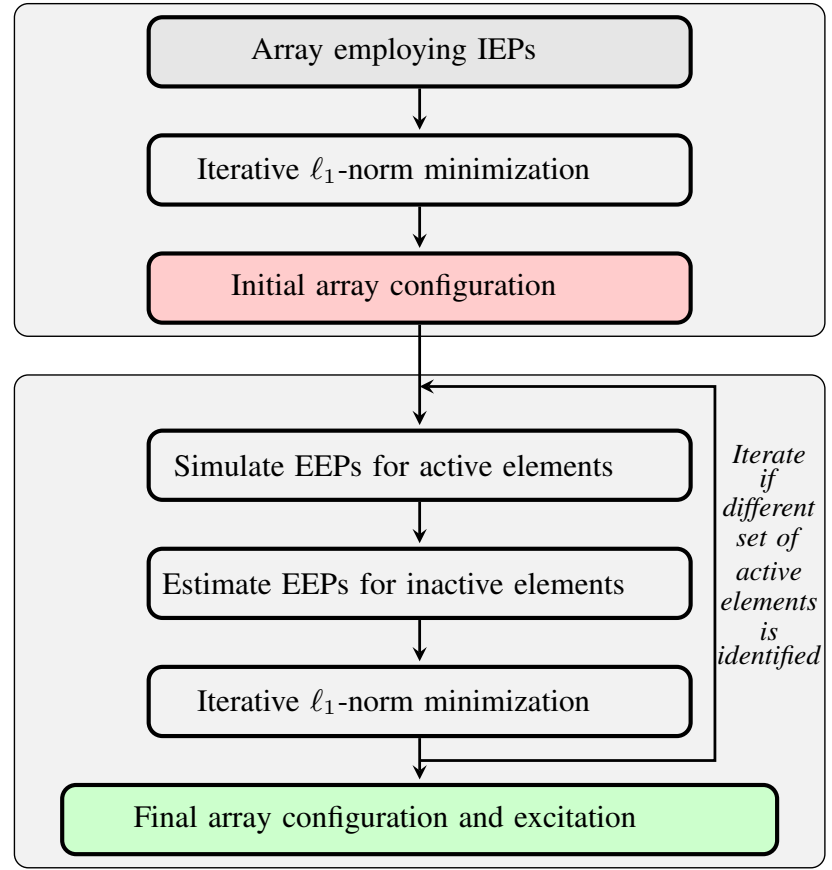

Fig. 1. Block diagram of the proposed iterative approach.

in the order of $-200 \mathrm{~dB}$, the distinction is clear and their removal from the actual array does not affect the total pattern.

The antenna MC is introduced in the second iterative phase, where a full-wave analysis is performed, but only for the active elements of the initial array layout. The isolated element patterns of the active antenna elements are now replaced by their simulated embedded element patterns (EEPs). Furthermore, the patterns of the inactive element patterns are estimated, i.e., by assuming a phase-shifted version of their nearest simulated embedded pattern of an active element ${ }^{2}$. In this manner, all the elements are ensured to assume embedded element patterns for the next iteration. Next, the iterative $\ell_{1}$ norm minimization algorithm is invoked, but now using the newly generated embedded element patterns for both active and inactive elements. If a different set of active elements has been identified, then another full-wave simulation is performed to update all the embedded element patterns as described above. This hybridized full-wave and $\ell_{1}$-norm minimization algorithm keeps iterating until the state of the active and inactive elements remain the same. Typically, only few MoM$\ell_{1}$ iterations are needed to reach convergence; for this purpose, the full-wave in-house developed CAESAR solver is used [13].

\section{NumericAl Results}

To examine the capabilities and performance figures of the herein proposed approach, two benchmark problems are considered, both employing relatively strongly coupled $y$ aligned $\lambda / 2$ dipoles arranged along the $x$-axis ( $H$-plane), at $z=\lambda / 4$ above a PEC ground plane (cf. Fig. 4). The first

\footnotetext{
${ }^{2}$ If needed, more sophisticated pattern interpolation techniques can be used to better estimate the embedded element patterns of inactive elements.
} 


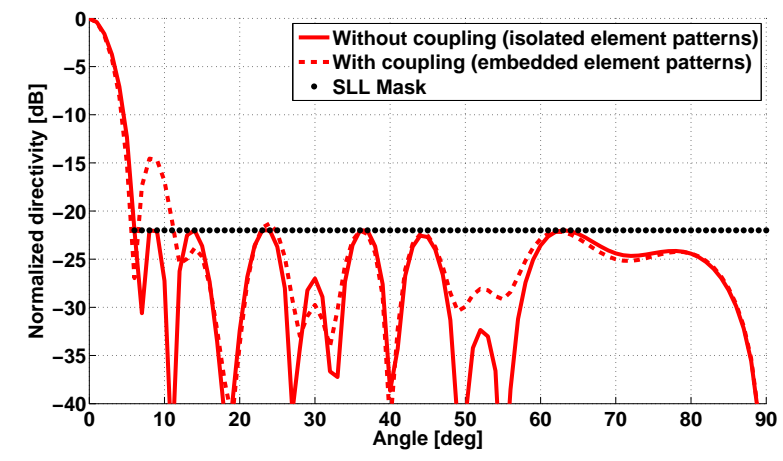

Fig. 2. Normalized directivity with and w/o MC effects for the initial array.

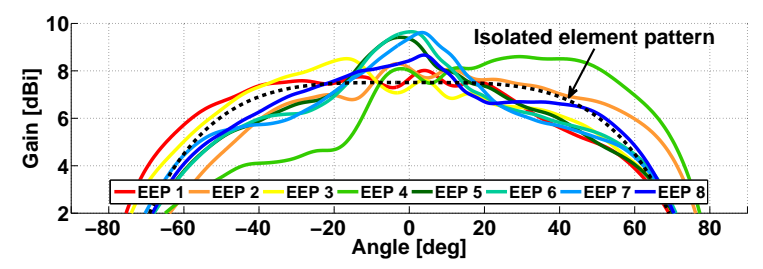

Fig. 3. Isolated and embedded element patterns for terminated case $(75 \Omega)$.

relatively simple case study demonstrates how our method compares to small sized arrays published in the literature, but now for arrays for which mutual coupling effects cannot be neglected. The second example considers a larger and more realistic array meeting the typically imposed SLL constraints used in space communication applications.

\section{A. Dipole Antenna Array of size $10 \lambda$}

We consider the problem of designing a broadside scanned array of aperture size $d=10 \lambda$ while imposing a constant SLL mask of $-20 \mathrm{~dB}$. This is a frequently used benchmark example for which the main beam is confined in the $|\theta| \leq 5.5^{\circ}$ $(|u| \leq 0.0965)$ region [12]. However, to compensate for the slightly higher element directivity with respect to the commonly employed isotropic radiator, the lower SLL of $-22 \mathrm{~dB}$ has been chosen. Furthermore, since we consider a broadside scanned array of identical antenna elements, a symmetric array layout will be synthesized.

As discussed above, the algorithm begins by assuming isolated element patterns and minimizes the number of highly

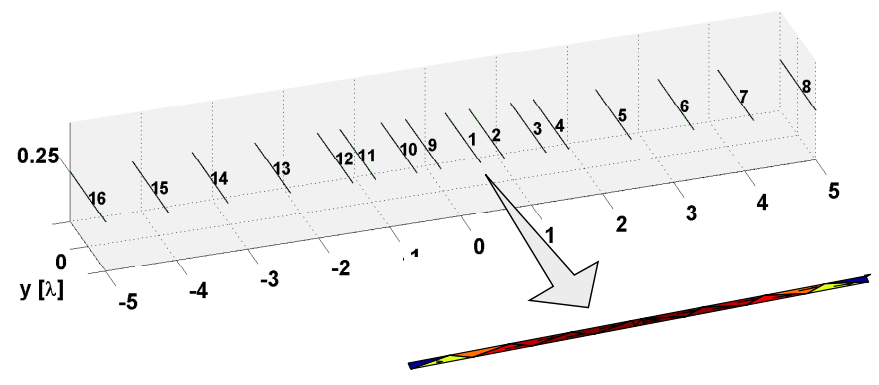

Fig. 4. Perspective view of meshed geometry for the initial array and detail of the current distribution on one element.

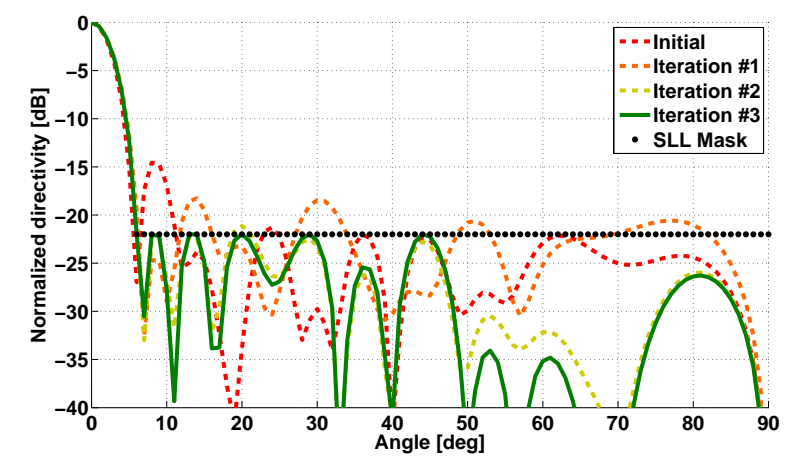

Fig. 5. Normalized directivity in the presence of MC for subsequent iterations.

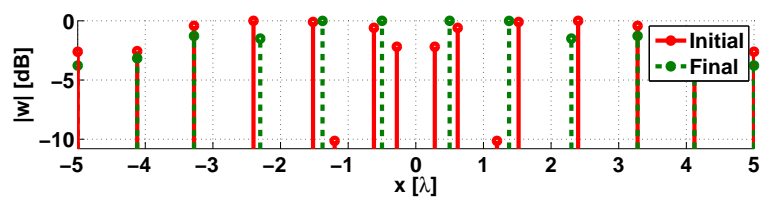

Fig. 6. Active elements positions and weights magnitude of initial and final array. Inactive elements (not shown) have magnitudes smaller than $-200 \mathrm{~dB}$.

weighted array elements, while satisfying the SLL constraints. Note that the initial solution is qualitatively equal to the common isotropic approximation. Accordingly, the initial array layout is simulated using a full-wave solver. The resulting array directivity patterns can be examined in Fig. 2, where the SLL degradation is seen to exceed $7 \mathrm{~dB}$ in proximity of the main lobe due to MC effects. The MC-included EEPs are plotted in Fig. 3, along with the isolated EEP for comparison. The array full-wave model is shown in Fig. 4.

The algorithm proceeds to re-optimize the array layout for the updated set of EEPs. The evolution of the array layout for each MoM- $\ell_{1}$ iteration can be tracked in Table I, where the element positions of half of the (symmetric) array are listed. Fig. 5 depicts the corresponding directivity patterns for each iteration. The initial and final element positions and weight magnitudes are shown in Fig. 6, where one can observe how the central and dense part of the array layout changes upon introducing MC effects. As can be concluded from Fig. 7(a), the array layout converges in just 3 iterations, while reducing the number of active elements from 16 to 12 , a compliant SLL is obtained, and the broadside directivity is barely compromised.

TABLE I

ELEMENT POSITIONS IN WAVELENGTHS FOR EACH ITERATION

\begin{tabular}{lcccccccc}
\hline Iteration & 1 & 2 & 3 & 4 & 5 & 6 & 7 & 8 \\
\hline Initial & 0.28 & 0.62 & 1.2 & 1.52 & 2.4 & 3.28 & 4.12 & 5 \\
Iter\#1 & & 0.5 & & 1.38 & 2.4 & 3.28 & 4.12 & 5 \\
Iter\#2 & & 0.5 & & 1.38 & 2.3 & 3.28 & 4.12 & 5 \\
Iter\#3 & & 0.5 & & 1.38 & 2.3 & 3.28 & 4.12 & 5 \\
\hline
\end{tabular}




\section{B. Dipole Antenna Array of size $120 \lambda$}

In space applications, the earth is often spot-illuminated from a satellite using narrow beams with stringent SLL constraints over the earth's surface. For geosynchronous satellites, typical aperture diameters of about $120 \lambda$ are required for beam widths in the order of $1^{\circ}$. Consider a linear array of such size, with a realistic SLL mask, such as the one from the ESA tender [14]; the main beam is confined within $0.325^{\circ}$ from broadside, the SLL constraint is $-27 \mathrm{~dB}$ from $0.795^{\circ}$ to $16^{\circ}$ (=earth subtending angle, as seen from the satellite) and $-10 \mathrm{~dB}$ outside this region (see Fig. 8). As can be seen, the initial array layout consists of 48 radiators and exhibits an inner mask SLL degradation of over $3 \mathrm{~dB}$ once the actual EEPs are used. In the subsequent iterations the MC-dependent EEPs are updated for each newly synthesized array; Fig. 9 shows the initial and final element positions for comparison. Fig. 7(b) shows that the number of elements reduces from 48 to 42 after just 4 iterations, while the SLL constraints are respected, at the cost of a modest reduction in the directivity at broadside.

\section{Conclusions}

A methodology has been proposed to synthesize maximally sparse antenna arrays in the presence of mutual coupling effects. The method operates by iteratively adjusting the array layout and excitations such as to minimize the number of elements while accounting for realistic EM effects while satisfying constraints on the maximum side-lobe level (SLL). The algorithm has been demonstrated for arrays of relatively strongly-coupled dipole antennas, for which the total number of active elements reduces by $10-20 \%$ from the initial (no coupling) to the last iteration (with coupling), while barely compromising its directivity. Despite the elements are strongly coupled, the method converges within a few iterations, thereby rendering the method computationally efficient. The penalty for not accounting for mutual coupling effects is manifested in the side lobes exceeding the maximum allowable SLL.

The method is currently being used for the design of large planar arrays of complex antenna elements analyzed through the numerically efficient CBFM. Ongoing research focusses on the inclusion of different antenna element types, multi-beam arrays, and to handle discrete amplitude level controls.

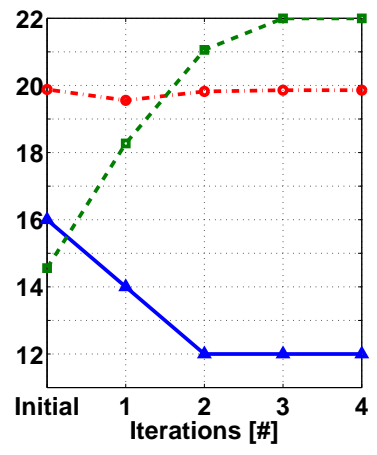

(a) $10 \lambda$ array.

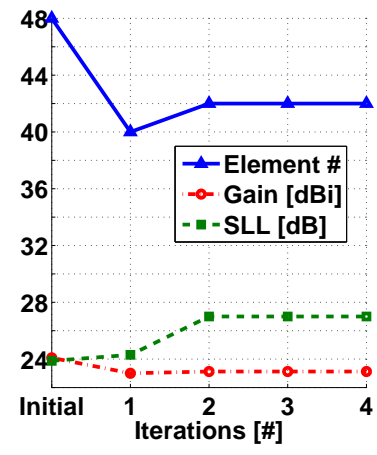

(b) $120 \lambda$ array.
Fig. 7. Number of elements, gain and SLL as a function of iteration count.

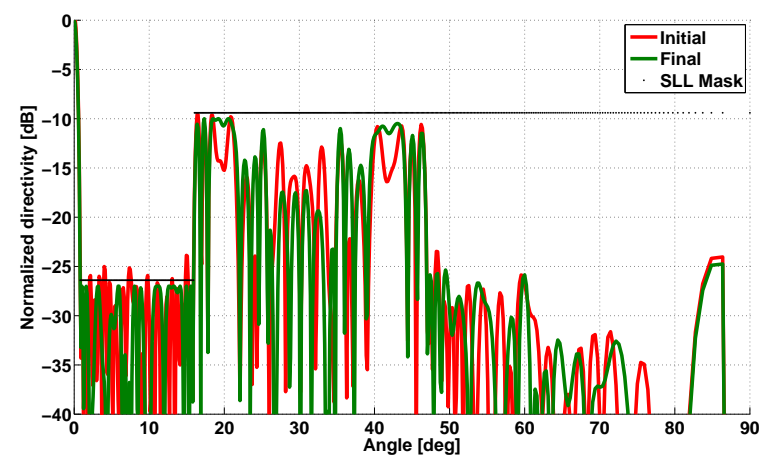

Fig. 8. Normalized directivity in the presence of MC effects for the initial and final synthesized arrays.

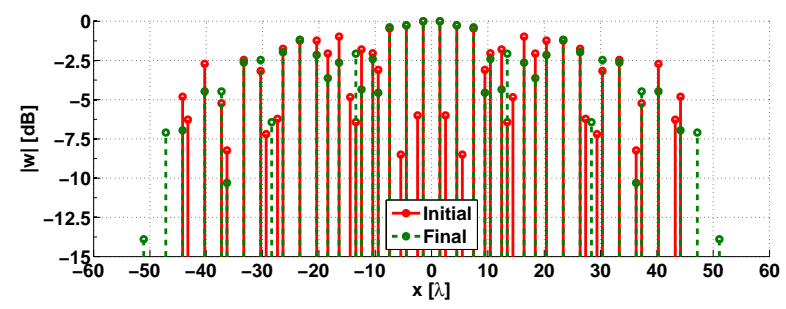

Fig. 9. Element positions and weight magnitudes for initial and final arrays

\section{REFERENCES}

[1] H. Unz, "Linear arrays with arbitrarily distributed elements," Antennas and Propagation, IRE Transactions on, vol. 8, no. 2, pp. 222-223, 1960.

[2] G. Toso and P. Angelletti, "Method of designing and manufacturing an array antenna," US patent filed as US0 211079 , Feb. 21, 2008.

[3] G. Oliveri, M. Donelli, and A. Massa, "Linear array thinning exploiting almost difference sets," IEEE Trans. Antennas Propag., vol. 57, no. 12, pp. 3800-3812, 2009

[4] R. L. Haupt, "Thinned arrays using genetic algorithms," IEEE Trans. Antennas Propag., vol. 42, no. 7, pp. 993-999, 1994.

[5] G. Oliveri, F. Caramanica, and A. Massa, "Hybrid ads-based techniques for radio astronomy array design," IEEE Trans. Antennas Propag., vol. 59, no. 6, pp. 1817-1827, 2009.

[6] Y. Liu, Z. Nie, and Q.-H. Liu, "Reducing the number of elements in a linear antenna array by the matrix pencil method," IEEE Trans. Antennas Propag., vol. 56, no. 9, pp. 2955-2962, 2008.

[7] O. Bucci, S. Perna, and D. Pinchera, "Advances in the deterministic synthesis of uniform amplitude pencil beam concentric ring arrays," IEEE Trans. Antennas Propag., vol. 60, no. 7, pp. 3504-3509, 2012.

[8] G. Oliveri, F. Caramanica, and A. Massa, "On the impact of mutual coupling effects on the psll performances of ads thinned arrays," Progress In Electromagnetics Research B, vol. 17, pp. 293-308, 2009.

[9] L. Cen, Z. L. Yu, and W. Ser, "Antenna array synthesis in presence of mutual coupling effect for low cost implementation," in Integrated Circuits, ISIC '09, 2009, pp. 360-363.

[10] T. Svantesson, "Modeling and estimation of mutual coupling in a uniform linear array of dipoles," in IEEE Int. Conf. on Acoustics, Speech, and Signal Processing, 1999, pp. 2961-2964.

[11] J. Corcoles and M. A. Gonzalez, "Efficient combined array thinning and weighting for pattern synthesis with a nested optimization scheme," Antennas and Propagation, IEEE Transactions on, vol. 60, no. 11, pp. 5107-5117, Nov 2012.

[12] G. Prisco and M. D'Urso, "Maximally sparse arrays via sequential convex optimizations," IEEE Antennas Wireless Propag. Lett., vol. 11, pp. 192-195, 2012.

[13] R. Maaskant, "Analysis of large antenna systems," Ph.D. dissertation, Eindhoven University of Technology, Eindhoven, 2010. [Online]. Available: http://alexandria.tue.nl/extra2/201010409.pdf

[14] ESA/ESTEC, "Innovative architectures for reducing the number of controls of multiple beam telecommunications antennas," Tender AO/15598/08/NL/ST, Jan. 2008. 